\title{
Automated Segmentation and Geometrical Modeling of the Tricuspid Aortic Valve in 3D Echocardiographic Images
}

\author{
Alison M. Pouch ${ }^{1}$, Hongzhi Wang ${ }^{2}$, Manabu Takabe ${ }^{1}$, Benjamin M. Jackson ${ }^{1,3}$, \\ Chandra M. Sehgal ${ }^{2}$, Joseph H. Gorman III ${ }^{1,3}$, Robert C. Gorman ${ }^{1,3}$, \\ and Paul A. Yushkevich ${ }^{2}$ \\ ${ }^{1}$ Gorman Cardiovascular Research Group \\ ${ }^{2}$ Department of Radiology \\ ${ }^{3}$ Department of Surgery, \\ University of Pennsylvania, Philadelphia, PA, USA
}

\begin{abstract}
The aortic valve has been described with variable anatomical definitions, and the consistency of 2D manual measurement of valve dimensions in medical image data has been questionable. Given the importance of imagebased morphological assessment in the diagnosis and surgical treatment of aortic valve disease, there is considerable need to develop a standardized framework for $3 \mathrm{D}$ valve segmentation and shape representation. Towards this goal, this work integrates template-based medial modeling and multi-atlas label fusion techniques to automatically delineate and quantitatively describe aortic leaflet geometry in 3D echocardiographic (3DE) images, a challenging task that has been explored only to a limited extent. The method makes use of expert knowledge of aortic leaflet image appearance, generates segmentations with consistent topology, and establishes a shape-based coordinate system on the aortic leaflets that enables standardized automated measurements. In this study, the algorithm is evaluated on 11 3DE images of normal human aortic leaflets acquired at mid systole. The clinical relevance of the method is its ability to capture leaflet geometry in 3DE image data with minimal user interaction while producing consistent measurements of 3D aortic leaflet geometry.
\end{abstract}

Keywords: medial axis representation, deformable modeling, multi-atlas segmentation, aortic valve, 3D echocardiography.

\section{Introduction}

The aortic valve regulates blood flow from the left ventricle to the ascending aorta and is an integral component of physiological cardiac function. In elderly populations, the valve is frequently affected by degenerative pathology, generally manifesting as stenosis or narrowing of the valve orifice. Aortic valve replacement is a commonly performed and preferred surgical treatment for aortic stenosis [1], and the emergence of transcatheter implantation has increased the patient population eligible for surgical intervention. For accurate prosthesis selection, these procedures require precise 
knowledge of aortic valve dimensions, which are conventionally specified by 2D manual measurement of echocardiographic or fluoroscopic images. However, agreement between multi-modal measurements has been equivocal [2-4], most likely because manually derived 2D metrics are limited in their capability to characterize complex 3D valvular geometry. Moreover, there is a surprising lack of consensus in the description of aortic valve geometry, particularly the annulus, which has been defined in markedly different ways [5]. Given the importance of quantitative metrics for diagnostics and treatment planning, there is need for a standardized framework for pre-operative image-based guidance of aortic valve surgery.

Limited work has been devoted to studying in vivo aortic valve geometry, most of which has been performed in ovine subjects [6] or in humans with manual 2D analysis of echocardiographic and multislice computed tomography (MSCT) images [24,7]. A few automated methods have been developed to segment the aortic root in MSCT data [8-9], but they do not delineate the aortic leaflets (also referred to as cusps). One proposed segmentation method parametrically represents both the 3D aortic root and leaflet geometry in MSCT images [10]. Thus, the algorithm presented here is one of few methods for automated 3D aortic leaflet shape analysis. The novelty of our work is in the generation of a volumetric segmentation from 3DE data that represents the aortic leaflets as structures with locally varying thickness. The advantage of using 3DE is that it facilitates 3D in vivo measurement of the aortic leaflets, is practical for routine use in the operating room, and does not require radiation exposure or contrast injection. Our method is a unique integration of multi-atlas segmentation and deformable medial modeling techniques, which incorporate expert knowledge of leaflet image appearance in the image analysis task. The algorithm is tested on systolic 3DE images acquired from normal subjects, a challenging application given the user-dependence of ultrasound image acquisition.

\section{Materials and Methods}

\subsection{Materials}

Electrocardiographically gated 3DE images of the aortic valve were acquired from 11 human subjects with normal aortic valve structure and function. The image data were acquired with the iE33 platform (Philips Medical Systems, Andover, MA) using a 2 to $7 \mathrm{MHz}$ matrix-array transesophageal transducer over several consecutive cardiac cycles. The image acquisition protocol did not specify constraints on image orientation or field of view. From each subject's data set, a 3DE image of the open aortic valve at mid systole was selected for analysis. The images were exported in Cartesian format with an approximate size of $224 \times 208$ x 208 voxels with nearly isotropic resolution ranging between 0.4 to $0.8 \mathrm{~mm}$.

\subsection{Manual Segmentation}

The 11 3DE images of the aortic valve were manually segmented in ITK-SNAP [11]. An expert observer identified the left coronary, non-coronary, and right coronary cusps, associating each with a separate label. In addition, three landmarks were 


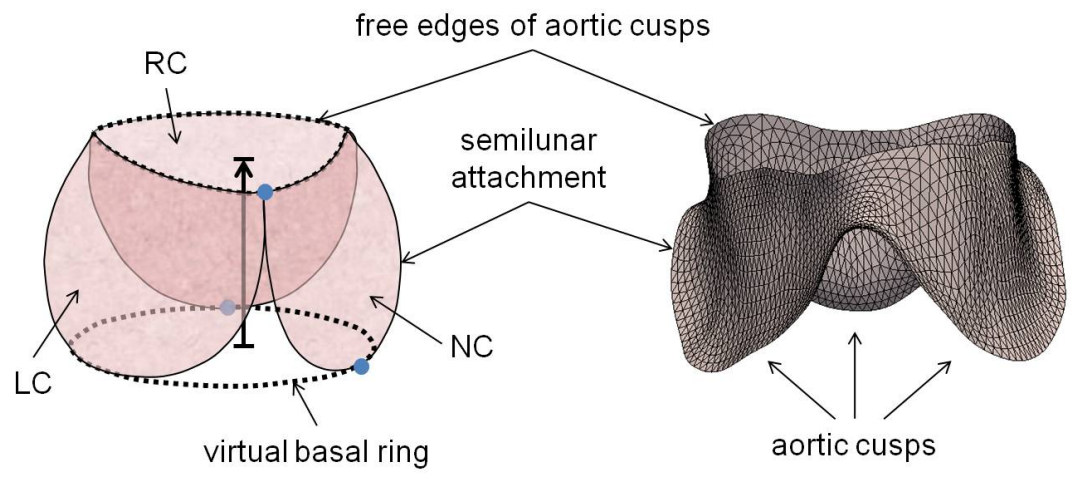

Fig. 1. (Left) Schematic of the aortic cusps at systole. The valve orifice is the area enclosed by the cusp free edges, and the virtual basal ring connects the basal attachments of the cusps. Valve height is the distance between the virtual basal ring and valve orifice, shown by the black arrow pointing in the direction of blood flow. Three manually identified landmarks are shown in blue. (Right) The triangulated medial template of the aortic cusps used to initialize deformable modeling. $(\mathrm{RC}=$ right coronary cusp, $\mathrm{LC}=$ left coronary cusp, $\mathrm{NC}=$ non-coronary cusp)

identified: the basal attachment of the non-coronary cusp, the basal attachment of the right coronary cusp, and the non-coronary commissure, illustrated in Fig. 1.

\subsection{Automated Image Analysis}

Automated segmentation and geometrical modeling of the aortic valve in a target image requires a deformable template of the aortic cusps and a set of probability maps, which assign each voxel in the target image a probability of having a given label. The deformable model is a medial axis representation of the aortic leaflets, and the probability maps are derived from multi-atlas joint label fusion, a technique that uses a set of expert-labeled 3DE images (atlases) of the aortic valve to generate a probabilistic segmentation of the target image. To represent aortic leaflet shape in the target image, the template is deformed to optimize a posterior probability in which the Bayesian likelihood is a function of the probability maps obtained by joint label fusion. In the segmentation process, multi-atlas label fusion and deformable medial modeling are complementary techniques: label fusion uses expert knowledge of aortic valve image appearance to estimate voxel-wise label probabilities in the target image, and deformable medial modeling ensures the topological consistency of the segmentations and identifies correspondences on different instances of the aortic valve. The combination of these capabilities facilitates standardized automated measurements of aortic cusp morphology in 3DE image data.

Multi-atlas Segmentation. Multi-atlas segmentation uses a small number of expertlabeled images, referred to as atlases, to make separate guesses at the segmentation of the target image. While the guesses are not accurate on their own, they are combined into a more robust segmentation result using a consensus-seeking scheme called label 
fusion. The present study makes use of joint label fusion, an extension of multi-atlas label fusion with spatially-varying weighted voting that reduces segmentation errors produced by redundancies in the atlas set. To perform multi-atlas segmentation, intensity-based registration is performed between all reference atlases and the target image. Registration consists of two stages. First, a global affine registration with six degrees of freedom is obtained by aligning the three landmarks identified during manual segmentation. Second, a B-spline free-form deformable registration is performed [12]. Cross-correlation is the similarity metric used for registration, and a Gaussian regularizer with sigma $=3$ is applied. Finally, each atlas is warped into the target image space to generate a candidate segmentation of the target image. A probabilistic consensus segmentation of the three aortic cusps is generated using the joint label fusion method described in [13].

Deformable Medial Modeling. The anatomical shape model used in this work is 3D continuous medial representation (cm-rep), which describes the geometry of an object in terms of its medial axis (or morphological skeleton) [14]. The medial axis is defined as the locus of centers of maximally inscribed balls of the object. In this framework, an object is parameterized by $\{\mathbf{m}, R\} \in \mathbb{R}^{3} \times \mathbb{R}^{+}$, where $\mathbf{m}$ is one or a combination of continuous medial manifolds and $R$ is a radial thickness field defined over the medial manifold(s). Each point $\mathbf{m}$ on the medial axis is associated with a scalar value $R$, the radius of the maximally inscribed ball centered at that point. The template-based approach to medial modeling involves first explicitly defining the object's skeletal topology in a deformable model or template. The template parameters $\{\mathbf{m}, R\}$ are modified to obtain the skeleton of an instance of the anatomic structure in a target image. Then the boundary of the structure, defined as the envelope of its maximally inscribed balls, is derived analytically from the medial axis according to the inverse skeletonization equations given in [14]. The medial model imposes a shape-based coordinate system on the anatomic structure that associates each point on the medial manifold $\mathbf{m}$ with one or more profiles of length $R$ that extend from the medial axis to unique surface patches on the object boundary.

In this work, the three aortic valve cusps are modeled as a single object with a nonbranching medial manifold. The manifold is discretely represented as a triangulated mesh, which can be sequentially Loop subdivided to a continuous limit. The medial template is generated using a method similar to that described in [15]. The 2D domain of the 3D medial mesh is homeomorphic to an annulus, in which the inner contour maps to the leaflet free edges and the outer contour maps to the semilunar attachments. The medial model of the aortic valve (Fig. 1) has 95 nodes on the leaflet free edges, 163 on the semilunar attachment curve, 1784 on the interior of the medial manifold, and 3824 total nodes on the model boundary. Each medial node is associated with five parameters: $\left(\mathrm{m}_{\mathrm{x}}, \mathrm{m}_{\mathrm{y}}, \mathrm{m}_{\mathrm{z}}, R, l\right)$, where $\left(\mathrm{m}_{\mathrm{x}}, \mathrm{m}_{\mathrm{y}}, \mathrm{m}_{\mathrm{z}}\right)$ refer to the 3D coordinates of a point on the medial axis and $R$ is the distance between that point and the leaflet's arterial and ventricular surfaces. The parameter $l$ refers to the leaflet label: $l=1$ is the left coronary cusp, $l$ $=2$ is the non-coronary cusp, and $l=3$ is the right coronary cusp. The medial template is initialized with a constant radial thickness: $R=2 \mathrm{~mm}$.

Given a target 3DE image $I(x)$ of the aortic valve, a deformable medial template $M$, and a set of probability maps $\left\{P^{l}(x): l=0,1,2,3\right\}$ obtained by multi-atlas joint 
label fusion, the medial template is deformed to minimize the negative log of the posterior probability $p(M \mid I)$, which is proportional to a likelihood function $p(I \mid M)$ and prior probability $p(M)$. The negative log of the Bayesian likelihood is a probability integral term given by

$$
-\log (p(I \mid M)) \sim 1-\frac{\left.\sum_{l} P^{l}(x)\right|_{M_{l}}}{I_{\text {volume }}},
$$

where $l$ indexes through each label, $x$ indexes through the image voxels, $I_{\text {volume }}$ is the target image volume, and $M_{l}$ represents the part of the model $M$ associated with label $l . \quad M_{0}$ refers to the exterior of the model (background). The Bayesian prior probability is modeled as a sum of validity constraints on medial geometry $\left(T_{\text {validity }}\right.$, detailed in [15]), and a regularization component that acts on the model's two medial edges:

$$
-\log (p(M)) \sim T_{\text {validity }}+\sum_{i}\left(1-\cos \left(\theta_{i}\right)\right)+\sum_{j}\left(1-\cos \left(\phi_{j}\right)\right)
$$

Here, $\theta_{i}$ is the angle between the outward normals of adjacent nodes $\mathbf{m}_{i}$ and $\mathbf{m}_{i+1}$ on the leaflet free edges, and $\phi_{j}$ is the angle between adjacent line segments formed by nodes $\mathbf{m}_{j-1}$ and $\mathbf{m}_{j}$ and nodes $\mathbf{m}_{j}$ and $\mathbf{m}_{j+1}$ on the leaflet attachment sites. In effect, the first regularization term encourages the leaflet free edges to point away from the ventricle into the aortic outflow tract, while the second term prevents rippling of the crown-shaped semilunar attachments during model deformation. The deformable model is rigidly initialized by aligning the three manually identified landmarks in the target image to those labeled on the deformable model. Optimization is performed by conjugate gradient descent.

\section{Results}

Segmentation and geometric modeling were evaluated in a leave-one-out crossvalidation experiment using manual image segmentation for comparison. The Dice overlap and mean boundary displacement metrics were used for comparison. The accuracy of each step in the segmentation process is given in Table 1, and an example of aortic leaflet segmentation is given in Fig. 2.

Table 1. The accuracy of each segementation step. The 95th percentile distance indicates the maximum distance of 95 percent of boundary points on the deformed model from the surface of the manual segmentation. The fourth column indicates the accuracy of model fitting directly to the manual segmentation.

\begin{tabular}{lcccc}
\hline & $\begin{array}{c}\text { Candidate } \\
\text { segmentation } \\
\text { (average) }\end{array}$ & $\begin{array}{c}\text { Consensus } \\
\text { segmentation }\end{array}$ & $\begin{array}{c}\text { Model-based } \\
\text { segmentation }\end{array}$ & $\begin{array}{c}\text { Model fitted } \\
\text { to manual } \\
\text { segmentation }\end{array}$ \\
\hline Dice overlap & $0.46 \pm 0.08$ & $0.74 \pm 0.3$ & $0.74 \pm 0.3$ & $0.84 \pm 0.02$ \\
Displacement (mm) & & & & \\
$\quad$ Mean & $0.9 \pm 0.4$ & $0.4 \pm 0.1$ & $0.5 \pm 0.1$ & $0.3 \pm 0.1$ \\
Maximum & $6.9 \pm 4.5$ & $3.5 \pm 0.7$ & $3.2 \pm 0.8$ & $2.7 \pm 0.7$ \\
95 $5^{\text {th }}$ percentile & $3.5 \pm 1.7$ & $0.9 \pm 0.3$ & $1.0 \pm 0.2$ & $0.7 \pm 0.1$ \\
\hline
\end{tabular}



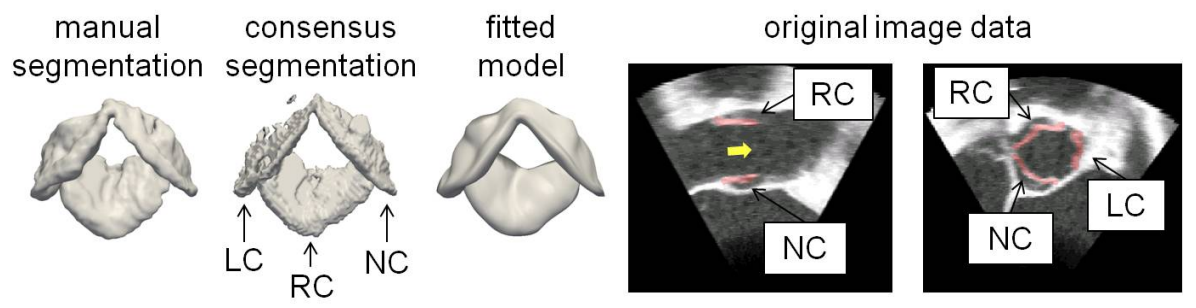

Fig. 2. A manual segmentation, consensus segmentation generated by label fusion, and fitted medial model. The model-based segmentation is shown in red (right). The yellow arrow points in the direction of blood flow. (LC, $\mathrm{RC}, \mathrm{NC}=$ left, right, and non-coronary cusps).

Table 2 lists a number of clinically relevant measurements that were automatically derived from the model-based segmentation. The measures were compared to those computed when the deformable model was fitted directly to the manual segmentation, the accuracy of which is presented in Table 1. For each measurement, the mean \pm standard deviation, the bias and limits of agreement given by Bland-Altman analysis, and the Pearson correlation coefficient are provided.

Table 2. Comparison of measurements obtained from the automated segmentation and from model fitting to the manual segmentation. (stdev $=$ standard deviation, $\mathrm{R}_{\text {coeff }}=$ Pearson coefficient, $\mathrm{VOA}=$ valve orifice area, $\mathrm{BROA}=$ basal ring orifice area, $\mathrm{VOD}_{\text {mean }}=$ mean valve orifice diameter, $\mathrm{BRD}_{\text {mean }}=$ mean basal ring diameter, $\mathrm{AH}=$ aortic valve height, $\mathrm{SA}_{\mathrm{LC}}=$ surface area of the left coronary cusp, $\mathrm{SA}_{\mathrm{NC}}=$ surface area of the non-coronary cusp, $\mathrm{SA}_{\mathrm{RC}}=$ surface area of the right coronary cusp, $R_{\text {mean }}=$ mean radial thickness).

\begin{tabular}{lccccc}
\hline & $\begin{array}{c}\text { Manual } \\
\text { (mean } \pm \text { stdev) }\end{array}$ & $\begin{array}{c}\text { Automated } \\
\text { (mean } \pm \text { stdev) }\end{array}$ & Bias & $\begin{array}{c}\text { Limits of } \\
\text { agreement }\end{array}$ & $\mathrm{R}_{\text {coeff }}$ \\
\hline VOA & $2.63 \pm 0.47 \mathrm{~cm}^{2}$ & $2.91 \pm 0.71 \mathrm{~cm}^{2}$ & $0.27 \mathrm{~cm}^{2}$ & -0.55 to $1.09 \mathrm{~cm}^{2}$ & 0.828 \\
BROA & $5.27 \pm 0.69 \mathrm{~cm}^{2}$ & $5.38 \pm 1.04 \mathrm{~cm}^{2}$ & $0.11 \mathrm{~cm}^{2}$ & -1.17 to $1.39 \mathrm{~cm}^{2}$ & 0.791 \\
$\mathrm{VOD}_{\text {mean }}$ & $18.2 \pm 1.7 \mathrm{~mm}$ & $19.2 \pm 2.3 \mathrm{~mm}$ & $1.0 \mathrm{~mm}$ & -1.7 to $3.7 \mathrm{~mm}$ & 0.808 \\
$\mathrm{BRD}_{\text {mean }}$ & $25.9 \pm 1.7 \mathrm{~mm}$ & $26.1 \pm 2.5 \mathrm{~mm}$ & $0.2 \mathrm{~mm}$ & -3.0 to $3.5 \mathrm{~mm}$ & 0.749 \\
$\mathrm{AH}$ & $19.0 \pm 2.3 \mathrm{~mm}$ & $17.9 \pm 1.6 \mathrm{~mm}$ & $-1.1 \mathrm{~mm}$ & -4.7 to $2.5 \mathrm{~mm}$ & 0.612 \\
$\mathrm{SA}_{\mathrm{LC}}$ & $3.93 \pm 0.92 \mathrm{~cm}^{2}$ & $3.47 \pm 0.61 \mathrm{~cm}^{2}$ & $-0.46 \mathrm{~cm}^{2}$ & -1.75 to $0.82 \mathrm{~cm}^{2}$ & 0.703 \\
$\mathrm{SA}_{\mathrm{NC}}$ & $3.61 \pm 0.45 \mathrm{~cm}^{2}$ & $3.36 \pm 0.60 \mathrm{~cm}^{2}$ & $-0.25 \mathrm{~cm}^{2}$ & -1.04 to $0.54 \mathrm{~cm}^{2}$ & 0.746 \\
$\mathrm{SA}_{\mathrm{RC}}$ & $3.87 \pm 0.53 \mathrm{~cm}^{2}$ & $3.78 \pm 0.69 \mathrm{~cm}^{2}$ & $-0.09 \mathrm{~cm}^{2}$ & -1.01 to $0.82 \mathrm{~cm}^{2}$ & 0.732 \\
$R_{\text {mean }}$ & $0.8 \pm 0.3 \mathrm{~mm}$ & $1.1 \pm 0.3 \mathrm{~mm}$ & $0.3 \mathrm{~mm}$ & -0.04 to $0.75 \mathrm{~mm}$ & 0.715 \\
\hline
\end{tabular}

\section{Discussion}

The proposed 3D aortic valve segmentation method combines the attractive properties of multi-atlas segmentation and deformable medial modeling to generate quantitatively descriptive representations of aortic leaflet anatomy in 3DE images. The algorithm begins by generating candidate segmentations of the target image, each produced by warping an individual atlas to the target image. Based on the metrics presented in 
Table 1, the candidates on their own poorly approximate the manual segmentation. Joint label fusion, a robust consensus-seeking scheme, produces a more accurate segmentation of the target image, indicated by a dramatic improvement in the Dice overlap and boundary displacement metrics. While multi-atlas label fusion can segment the target image without the use of deformable modeling, it does not guarantee the topological consistency of different instances of the aortic leaflets. This is evidenced by the extraneous artifact and leaflet hole that can be seen in Fig. 2. Nor does multiatlas segmentation establish correspondences on different aortic valve segmentations, which makes it difficult to automatically compute standardized measurements of aortic leaflet geometry. Deformable medial modeling complements label fusion by mapping each segmented aortic valve to a common shape-based coordinate system, imposing validity and regularization constraints during Bayesian optimization, and by explicitly encoding the topology of the aortic cusps in the deformable model. With the probability maps generated by joint label fusion, the cm-rep can accurately capture leaflet shape, which is demonstrated by a mean boundary displacement from the manual segmentation on the order of 1 voxel.

Since the measurements presented in Table 2 could not be manually computed with the software used for manual tracing, the measurements derived from the automated segmentation were compared to those obtained by fitting the model directly to the manual segmentation. The advantage of such a comparison is that all measurements are made in 3D using the same software and consistent anatomical definitions. The disadvantage is that the comparison may be affected by the error in model fitting to the manual segmentation presented in Table 1 . The results in Table 2 demonstrate consistency between the measurements derived from the automated and manual segmentations. Our measurements of $\mathrm{BRD}_{\text {mean }}$, BROA, and VOA are comparable to analogous measurements in normal subjects made by MSCT planimetry in [2] and [7].

Unlike most existing methods for aortic valve assessment, the only requisite user interaction of the algorithm is the identification of three landmarks, which are used to initialize deformable registration and model fitting. Although the algorithm has not yet been tested on diseased subjects, the goal of the study is to demonstrate the feasibility of applying the method to a normal population before extending the methodology to pathological assessment. Given our previous success in using cm-rep to model both normal and diseased mitral valves [15] and the parallels between mitral and aortic valve disease processes, we expect the proposed method to characterize normal and pathological aortic cusp geometry equally well. The development of this automated technique is a step towards creating a practical, informative tool for preoperative assessment of patient-specific aortic valve morphology.

Acknowledgement. This research was supported by an American Heart Association pre-doctoral fellowship (10PRE3510014) and the National Institutes of Health: HL063954, HL073021, and HL103723 from the NHLBI; AG037376 from the NIA; and EB014346 from the NIBIB. 


\section{References}

1. Bonow, R.O., Carabello, B.A., Chatterjee, K., de Leon Jr., A.C., Faxon, D.P., Freed, M.D., Gaasch, W.H., Lytle, B.W., Nishimura, R.A., O'Gara, P.T., O’Rourke, R.A., Otto, C.M., Shah, P.M., Shanewise, J.S., Smith Jr., S.C., Jacobs, A.K., Adams, C.D., Anderson, J.L., Antman, E.M., Fuster, V., Halperin, J.L., Hiratzka, L.F., Hunt, S.A., Nishimura, R., Page, R.L., Riegel, B.: ACC/AHA 2006 Guidelines for the Management of Patients with Valvular Heart Disease. J. Am. Coll. Cardiol. 48(3), e1-e148 (2006)

2. Akhtar, M., Tuzcu, E.M., Kapadia, S.R., Svensson, L.G., Greenberg, R.K., Roselli, E.E., Halliburton, S., Kurra, V., Schoenhagen, P., Sola, S.: Aortic Root Morphology in Patients Undergoing Percutaneous Aortic Valve Replacement: Evidence of Aortic Root Remodeling. J. Thorac. Cardiovasc. Surg. 137(4), 950-956 (2009)

3. Messika-Zeitoun, D., Serfaty, J.M., Brochet, E., Ducrocq, G., Lepage, L., Detaint, D., Hyafil, F., Himbert, D., Pasi, N., Laissy, J.P., Iung, B., Vahanian, A.: Multimodal Assessment of the Aortic Annulus Diameter: Implications for Transcatheter Aortic Valve Implantation. J. Am. Coll. Cardiol. 55(3), 186-194 (2010)

4. Moss, R.R., Ivens, E., Pasupati, S., Humphries, K., Thompson, C.R., Munt, B., Sinhal, A., Webb, J.G.: Role of Echocardiography in Percutaneous Aortic Valve Implantation. JACC Cardiovasc. Imaging. 1(1), 15-24 (2008)

5. Anderson, R.H.: The Clinical Anatomy of the Aortic Root. Heart 84, 670-673 (2000)

6. Dagum, P., Green, G.R., Nistal, F.J., Daughters, G.T., Timek, T.A., Foppiano, L.E., Bolger, A.F., Ingels Jr., N.B., Miller, D.C.: Deformational Dynamics of the Aortic Root: Modes and Physiologic Determinants. Circulation 100(19 suppl.), II54-II62 (1999)

7. Tops, L.F., Wood, D.A., Delgado, V., Schuijf, J.D., Mayo, J.R., Pasupati, S., Lamers, F.P., van der Wall, E.E., Schalij, M.J., Webb, J.G., Bax, J.J.: Noninvasive Evaluation of the Aortic Root with Multislice Computed Tomography Implications for Transcatheter Aortic Valve Replacement. JACC Cardiovasc. Imaging. 1(3), 321-330 (2008)

8. Wang, Q., Book, G., Contreras Ortiz, S.H., Primiano, C., McKay, R., Kodali, S., Sun, W.: Dimensional Analysis of Aortic Root Geometry during Diastole using 3D Models Reconstructed from Clinical 64-Slice Computed Tomography Images. Cardiovasc. Eng. Technol. 2(4), 324-333 (2011)

9. Zheng, Y., John, M., Liao, R., Nottling, A., Boese, J., Kempfert, J., Walther, T., Brockmann, G., Comaniciu, D.: Automatic Aorta Segmentation and Valve Landmark Detection in C-arm CT for Transcatheter Aortic Valve Implantation. IEEE Trans. Med. Imaging. 31(12), 2307-2321 (2012)

10. Ionasec, R.I., Tsymbal, A., Vitanovski, D., Georgescu, B., Zhou, S.K., Navab, N., Comani-ciu, D.: Shape-Based Diagnosis of the Aortic Valve. In: Proc. SPIE Med. Imaging, vol. 7259 (2009)

11. Yushkevich, P.A., Piven, J., Hazlett, H.C., Smith, R.G., Ho, S., Gee, J.C., Gerig, G.: UserGuided 3D Active Contour Segmentation of Anatomical Structures: Significantly Improved Efficiency and Reliability. Neuroimage 31(3), 1116-1128 (2006)

12. Tustison, N.J., Avants, B.B., Gee, J.C.: Directly Manipulated Free-Form Deformation Image Registration. IEEE Trans. Image Process. 18(3), 624-635 (2009)

13. Wang, H., Suh, J.W., Das, S., Pluta, J., Craige, C., Yushkevich, P.: Multi-Atlas Segmentation with Joint Label Fusion. IEEE Trans. Pattern Anal. Mach. Intell. 35(3), 611-623 (2013)

14. Yushkevich, P.A., Zhang, H., Gee, J.C.: Continuous Medial Representation for Anatomical Structures. IEEE Trans. Med. Imaging. 25(12), 1547-1564 (2006)

15. Pouch, A.M., Yushkevich, P.A., Jackson, B.M., Jassar, A.S., Vergnat, M., Gorman III, J.H., Gorman, R.C., Sehgal, C.M.: Development of a Semi-Automated Method for Mitral Valve Modeling with Medial Axis Representation using 3D Ultrasound. Med. Phys. 39(2), 933-950 (2012) 